\title{
HYPOPHOSPHATASIA IN THE NEWBORN
}

\author{
BY \\ P. D. LAPATSANIS and R. MCLAREN TODD \\ From the Department of Child Health, University of Liverpool and the Liverpool Maternity Hospital
}

(RECEIVED FOR PUBLICATION AUGUST 9, 1962)

Rathbun (1948) first described the syndrome of hypophosphatasia as a definite entity, although previous authors had described patients now thought to be examples of this syndrome. This disorder has also been called rickets with deficiency of alkaline phosphatase (Sobel, Clark, Fox and Robinow, 1953; Schlesinger, Luder and Bodian, 1955) and osteodysmetamorphosis foetalis (Engfeldt and Zetterström, 1954).

The clinical manifestations of hypophosphatasia vary according to the time of onset. In severely affected babies the features are present at birth or before birth, and include a globular bonedeficient skull, skeletal deformities, renal lesions and defective pulmonary ventilation; in less severely affected infants, generalized skeletal deformities, anorexia, vomiting, hypotonia and irritability develop during the first six months of life; and in mildly affected patients, premature loss of deciduous teeth and bowing of the long bones may be the presenting features.

The incidence of hypophosphatasia, based upon four confirmed cases in unrelated families seen at the Hospital for Sick Children, Toronto (Fraser, 1957 ), is about 1 in 100,000 births. This case report is concerned with the first newborn baby with hypophosphatasia born in the Liverpool Maternity Hospital where about 3,000 babies are born each year.

\section{Case Report}

A multigravida aged 41 years was first seen in the antenatal clinic of the Liverpool Maternity Hospital on October 10, 1961. This was her fifth pregnancy and the expected date of confinement was March 17, 1962.

Previous Obstetrical History. 1942: Normal delivery at home of a full-term, live female infant. 1945: Normal delivery at home of a premature baby, 32 weeks, died at the age of 3 days; no autopsy report. 1946: Normal delivery at 32 weeks in St. Catherine's Hospital, Birkenhead, of baby who died at the age of 3 days; no autopsy report available. 1947: Normal delivery at 32 weeks in the same hospital, but the baby died 24 hours after birth; autopsy was not carried out.
Her blood group was A Rh negative and her husband's blood group was $\mathrm{O} R \mathrm{R}$ positive (probable phenotype CDe/cde).

Rhesus antibodies were demonstrated in albumin and saline on October 10, 1961; haemoglobin was $94 \%$ and chest radiograph was negative. Regular antenatal care was carried out and the pregnancy progressed normally until January 2, 1962, when she was admitted for investigation of hydramnios. A sample of liquor amnii obtained at 30 weeks contained $0.19 \mathrm{mg} . / 100 \mathrm{ml}$. bilirubin. Urine showed a positive Friedman test at a dilution of 1 in 100; the output of chorionic gonadotrophins was thus at least 4,300 i.u. in 24 hours. Radiograph of abdomen (Fig. 1) showed bone abnormalities, ? achondroplasia.

The patient went into labour spontaneously at the 38th week and was delivered of a live female infant weighing $5 \mathrm{lb} .4 \mathrm{oz}$. $(2 \cdot 38 \mathrm{~kg}$.).

The Baby. Her general condition was extremely poor, cyanosis was marked and respirations were established following intubation. No clinical jaundice was present, but the liver was enlarged 2 in. below the right costal margin and the spleen 1 in. below the left costal margin. The skull bones were soft anteriorly and deficient in the occipital region. The heart was normal in size, a grade 3 systolic murmur was heard over the praecordium and femoral pulses were present. Cord blood showed haemoglobin $106 \%(15.59 \mathrm{~g} . / 100 \mathrm{ml}$.$) , serum bilirubin$ $2.5 \mathrm{mg} . / 100 \mathrm{ml}$. and positive Coombs test. A radiograph revealed gross skeletal deformities of the long bones (Fig. 2) and skull (Fig. 3) typical of congenital hypophosphatasia. The results of alkaline phosphatase estimations of the family were as follows: baby, 1.5; mother, 10; father, 9; sister (aged 20), 7 King-Armstrong units.

Autopsy. The baby died two hours after birth and the chief abnormal findings at autopsy were as follows:

Cardiovascular system: Heart muscle and valves normal; interatrial septum and ductus arteriosus patent; vessels and coronary arteries normal.

Respiratory system: Pleurae and air passages normal; lungs showed only slight expansion.

Central nervous system: Bones of the cranial vault were very small and widely separated by membranes. Those of the base of the skull were thin and soft, but of normal form. The tentoria were small. The venous sinuses 


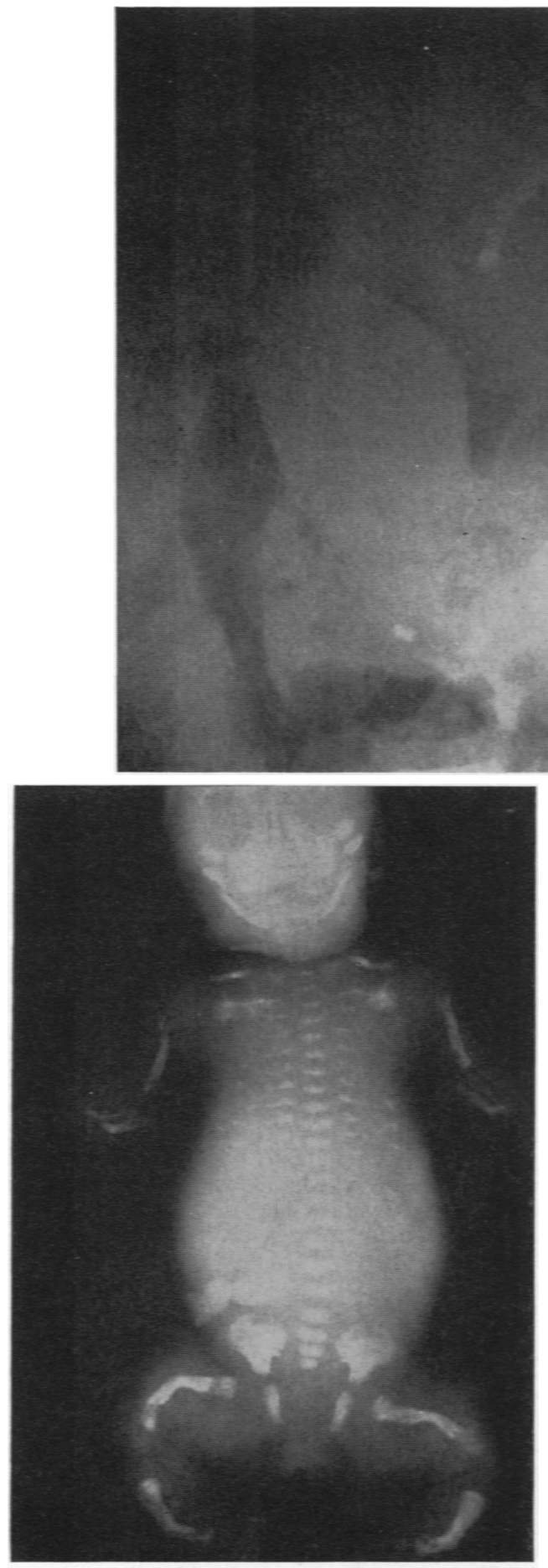

Fig. 2.

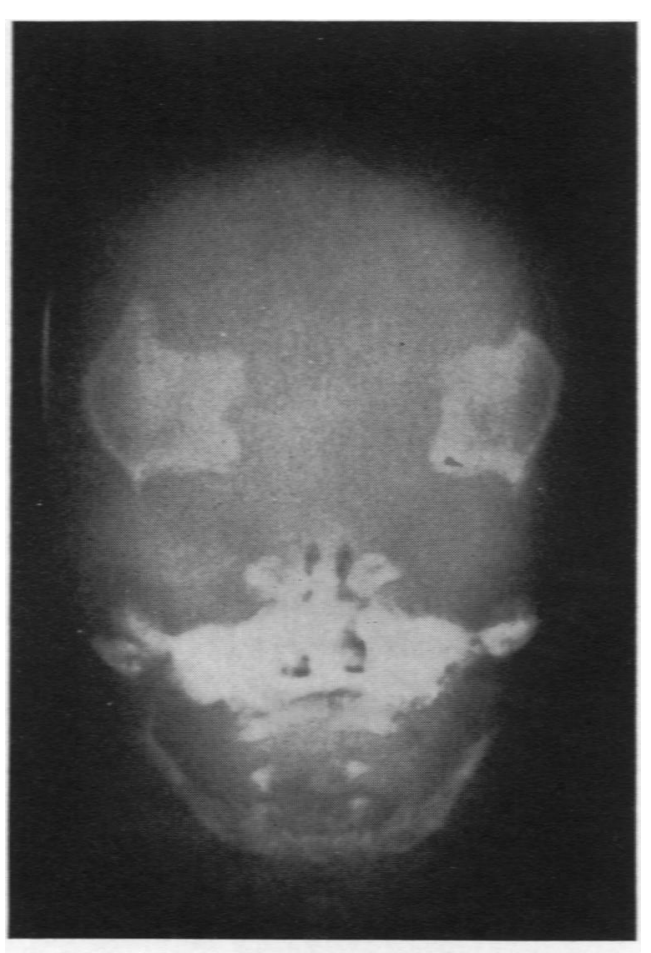

○े

음

흘. 
and brain itself appeared normal and no staining of the basal nuclei was found.

Skeletal system: The lower end of the shaft of the femur appeared moderately expanded. The cut surface showed an irregular epiphyseal line. Microscopically this region consists largely of uncalcified osteoid arranged in irregular masses between which are interspersed islands of hydropic and degenerating cartilage cells. There is abundant erythropoietic and vascular connective tissue. The lower part of the shaft is made up mainly of trabecular bone divided transversely by narrow bands of partly calcified osteoid. The lateral aspect of the cortex of the mid-shaft region is made up of narrow plates of bone with prominent osteoid seams. The medial aspect is made up of uncalcified osteoid tissue extending upwards to the capital epiphysis. There is an angulation deformity in the mid-shaft region composed of dense, fibrous tissue with uncalcified osteoid on either side. The small plaque of bone in the region of the parietal protuberance is made up of plates of normally calcified bone with wide osteoid seams. At the edge of the plaque these merge with plates of uncalcified osteoid which make up the rest of the parietal bone.

Urinary system: Both kidneys were normal in size. pattern and surface and microscopically. Pelves. ureters and bladder were normal.

Placenta: Weighed $590 \mathrm{~g}$. and appeared grossly normal.

\section{Discussion}

Hypophosphatasia may be confused with other generalized disorders of bone such as achondroplasia, osteogenesis imperfecta and hyperparathyroidism; but in these conditions, although the appearances of the long bones may be similar, there is not such gross deficiency of skull bone formation as in hypophosphatasia.

Because of the known recessive method of inheritance, it is possible once characteristic radiological changes have been observed in one baby to detect hypophosphatasia before birth in a subsequent pregnancy (McCance, Fairweather, Barrett and Morrison, 1956). Furthermore, carriers among parents and relatives may be detected by finding low or borderline serum alkaline phosphatase levels. Abnormally low alkaline phosphatase levels may also be found in scurvy and hypothyroidism.

McCance, Morrison and Dent (1955) and Fraser, Yendt and Christie (1955) have shown that phosphoethanolamine is excreted in the urine of patients with hypophosphatasia. It is probable that this substance acts as a substrate for alkaline phosphatase

\footnotetext{
FIG. 1.-Antenatal radiograph showing deformities of limb bones and lack of calcification of skull.

FrG. 2.-Generalized skeletal deformities.

FKG. 3.-Skull radiograph showing patchy ossification of frontal bones. moderately well-cakified base and marked decalcification of the other skull bones.
}

and in hypophosphatasia there is an accumulation of unused phospho-ethanolamine in the blood, and this is excreted in the urine. It was not possible to test for phospho-ethanolamine in our patient because she died within two hours of birth.

The histological changes in the long bones are characteristic; at the growing ends of the bones there is an absence of calcification and also an excessive production and disordered arrangement of cartilage cells and osteoid. These changes are similar to those seen in rickets. Renal changes have also been reported and consist in deposition of calcium in the renal tubular epithelium and in the surrounding interstitial tissue.

The prognosis seems to depend mainly on three factors, the age at the onset of symptoms, the degree of phosphatase deficiency and the severity of the associated renal and pulmonary lesions.

In the reported case the onset of the disease was in foetal life, the phosphatase deficiency was marked but there was no gross renal lesion. If the disease is present at birth or shortly afterwards the cause of death is frequently respiratory, due to an inability to ventilate the lungs adequately. McCance et al. (1956) and Kellsey (1962) suggested that this respiratory inadequacy was due to marked shortening of the ribs, which reduces the total thoracic cavity to about $25 \%$.

In the present case, the clinical picture and autopsy findings of only slight lung expansion were consistent with death from respiratory insufficiency.

\section{Summary}

The clinical, radiological and autopsy findings of a newborn baby suffering from hypophosphatasia are described.

We wish to thank Mr. J. Hamilton and Mr. C. H. de Boer for permission to publish this case.

\section{REFERENCES}

Engfeldt. B. and Zetterström. R. (1954). Osteodysmetamorphosis fetalis. A clinical-pathological study of a congenital skeletal disease with retarded growth, hypophosphatasemia and renal damage. J. Pediat., 45, 125.

Fraser, D. (1957). Hypophosphatasia. Amer. J. Med.. 22730

-. Yendt. E. R. and Christie. F. H. E. (1955). Metabolic abnor malities in hypophosphatasia. Lancet, 1, 286.

Kellsey. D. C. (1962). Hypophosphatasia and congenital bowing of the long bones. J. Amer. med. Ass., 179, 187 .

McCance, R. A., Fairweather, D. V. I., Barrett, A. M. and Morrison. A. B. (1956). Genetic, clinical. biochemica! and pathological features of hypophosphatasia. Quart. J. Med.. 25, 523. Morrison. A. B. and Dent, C. E. (1955). The excretion of phosphoethanolamine and hypophosphatasia. Lancet. 1. 131

Rathbun. J. C. (1948). Hypophosphatasia. Amer. J. Dis. Child. 75. 822 .

Schlesinger, B.. Luder, J. and Bodian, M. (1955). Rickets with alkaline phosphatase deficiency: an osteoblastic dysplasia. Arch. Dis. Childh.. 30. 265.

Sobel. E. H., Clark, C. L., Fox, R. P. and Robinow, M. (1953). Rickets. deficiency of "alkaline" phosphatase activity and premature loss of teeth in childhood. Pediatrics, 11. 309. 